\title{
ICA Mixtures Applied to Ultrasonic Nondestructive Classification of Archaeological Ceramics
}

\author{
Addisson Salazar and Luis Vergara \\ Grupo de Tratamiento de Señal, Instituto de Telecomunicaciones y Aplicaciones Multimedia, iTEAM, \\ Universidad Politécnica de Valencia, Camino de Vera s/n, 46022 Valencia, Spain \\ Correspondence should be addressed to Luis Vergara, lvergara@dcom.upv.es
}

Received 23 December 2009; Revised 7 May 2010; Accepted 7 June 2010

Academic Editor: João Manuel R. S. Tavares

Copyright ( $\odot 2010$ A. Salazar and L. Vergara. This is an open access article distributed under the Creative Commons Attribution License, which permits unrestricted use, distribution, and reproduction in any medium, provided the original work is properly cited.

\begin{abstract}
We consider a classifier based on Independent Component Analysis Mixture Modelling (ICAMM) to model the feature jointprobability density. This classifier is applied to a challenging novel application: classification of archaeological ceramics. ICAMM gathers relevant characteristics that have general interest for material classification. It can deal with arbitrary forms of the underlying probability densities in the feature vector space as nonparametric methods can do. Mutual dependences among the features are modelled in a parametric form so that ICAMM can achieve good performance even with a training set of relatively small size, which is characteristic of parametric methods. Moreover, in the training stage, ICAMM can incorporate probabilistic semisupervision (PSS): labelling by an expert of a portion of the whole available training set of samples. These properties of ICAMM are well-suited for the problem considered: classification of ceramic pieces coming from four different periods, namely, Bronze Age, Iberian, Roman, and Middle Ages. A feature set is obtained from the processing of the ultrasonic signal that is recorded in through-transmission mode using an ad hoc device. A physical explanation of the results is obtained with comparison with classical methods used in archaeology. The results obtained demonstrate the promising potential of ICAMM for material classification.
\end{abstract}

\section{Introduction}

Determining the historical period of archaeological ceramic shards is important for many archaeological applications, particularly to reconstruct human activities of the past. In fact, the standardization of an efficient and nondestructive testing (NDT) method for ceramic characterization could become an important contribution for archaeologists. Chemical, thermoluminescence, and other analyses have shown to measure the age of ceramics accurately, but they are expensive, time-consuming and involve some destruction of the analyzed pieces [1]. Relative dating by comparison with ceramic collections is nondestructive but very inaccurate [1].

Ultrasound has been used in archaeological applications such as ocean exploration to detect wrecks, imaging of archaeological sites, and cleaning archaeological objects [24]. In this paper, we consider a method to sort archaeological ceramic shards based on ultrasonic nondestructive evaluation. This method aims to be economic, fast, precise, and innocuous for the ceramic pieces. It consists of three steps: measuring by the through-transmission technique, extracting features from the measured ultrasonic signals, and classifying the feature set in classes corresponding to historic or protohistoric periods.

The estimation of the chronological period of an archaeological fragment is not a straightforward work, especially if we consider that the fragment might be moved from its context of origin due to migrations, wars, or trade exchange, and so forth. In addition, some external features used for classification of archaeological objects, such as particular shapes and decorations, might be not evident in the fragments, and thus these aspects would not provide information for a correct classification of the fragments.

Through-transmission was selected because the ceramic produces large attenuation to the propagation of ultrasound, 
so the pulse-echo technique cannot be implemented at the required operating frequency. Time, frequency, and statistical features (to be described later) were extracted using standard signal processing techniques. The characteristics of the classification problem offer a good case study for testing advanced classifiers, like those based on modelling the underlying statistical densities of the feature space as mixtures of independent component analyzers.

In consequence, we dedicate Section 2 to presenting the ultrasound through-transmission model from a linear system perspective and to defining the selected features. Then, in Section 3 we present the rationale for these classifiers and describe them based on mixtures of independent component analyzers. Section 4 presents the experiments and the results obtained in the sorting of ceramic pieces from four different periods: Bronze Age, Iberian, Roman, and Middle Ages. Section 5 presents the conclusions and future line of work.

We reported some preliminary results related to this archaeological application which was presented in conference [5]. The following significant new contributions are presented in this paper: rationale and selection of new ultrasonic features; use of a classifier that is based on probabilistic semisupervision of independent component analyzers (ICA) mixture models that are suitable for handling expert uncertainty; implementation of an ad hoc device designed to avoid the uncontrolled conditions of a totally manual measurement procedure; and demonstration of physical interpretation of the results obtained by the proposed method in comparison with classical methods used in archaeology. Therefore, this work provides the foundations to implement a practical method to complement or even replace some of the destructive and time-consuming techniques that are currently employed in archaeology.

\section{Through-Transmission Model and Features Definition}

A simplified model of ultrasonic through-transmission analysis is to consider that the recorded signal is the convolution of the material reflectivity with a linear time-varying system (LTV) (see Figure 1). The variant impulse response of the LTV is the injected ultrasonic pulse travelling through the material, which bears the effects of attenuation and dispersion that affect both its amplitude and frequency content. Actually, some nonlinearity may be incorporated into this simple model in some specific cases; however, in general, the linear assumption is adequate for a large number of situations, or is at least enough to be able to obtain practical solutions yielding reasonable performance. Thus, the received signal $x(t)$ looks similar to the one shown in Figure 1.

If we consider that $x(t)$ is a realization of a nonstationary stochastic process $\{\tilde{x}(t)\}$ having instantaneous power spectral density $P_{x}(f, t)$, different "ultrasonic signatures" us $(t)$ can be computed like those included in the following
Centroid frequency $\left(f_{c}\right)$ :

$$
\operatorname{us}(t)=f_{c}(t)=\frac{\int_{f_{1}}^{f_{2}} f \cdot P_{x}(f, t) d f}{\int_{f_{1}}^{f_{2}} P_{x}(f, t) d f} .
$$

Maximum frequency $\left(f_{\max }\right)$ :

$$
\operatorname{us}(t)=f_{\max }(t)=\underbrace{\max }_{f} P_{x}(f, t) .
$$

Bandwidth (BW):

$$
\operatorname{us}(t)=\operatorname{BW}(t)=\frac{\int_{f_{1}}^{f_{2}}\left(f-f_{c}(t)\right)^{2} \cdot P_{x}(f, t) d f}{\int_{f_{1}}^{f_{2}} P_{x}(f, t) d f} .
$$

Maximum frequency amplitude $\left(A f_{\max }\right)$ :

$$
\operatorname{us}(t)=\max P_{x}(f, t) .
$$

These signatures are measures of the spectral content variations that are affected by the ultrasonic pulse travelling inside the material. They can be estimated by means of wellknown smoothing techniques of time-frequency spectral analysis [6].

From us $(t)$, we can obtain features in different forms. For example, the time average value $\left(1 /\left(t_{1}-t_{0}\right)\right) \int_{t_{0}}^{t_{1}} \mathrm{us}(t) d t$ or the instantaneous value at one particular time instant us $\left(t_{0}\right)$ can be elements of the feature vector in the observation space.

Other time-domain features, such as the parameters $A$ and $\beta$ corresponding to an exponential model of the signal attenuation $\hat{x}(t)=A e^{-\beta t}$ or the total signal power received $P=\int_{0}^{T}|x(t)|^{2} d t / T$, are also possible to complement the frequency-domain features.

More features can be defined considering special conditions of the through-transmission model. For example, higher-order statistics can be used to detect the possible degree of non-Gaussianity of the reflectivity by measuring higher-order moments of the received signal like $H O M=$ $E\left[x\left(n T_{s}\right) \cdot x\left((n-1) T_{s}\right) \cdot x\left((n-2) T_{s}\right)\right][7]$, where $E[\cdot]$ means statistical expectation and $1 / T_{s}$ is the sampling frequency. Departures from the linear model of Figure 1 can be tested in different forms, for example, using the so-called timereversibility [8], which is defined by $T R=E\left[(d x(t) / d t)^{3}\right]$.

\section{Independent Component Analysis Mixture Model}

Let us consider a probabilistic classification context where some selected features are organized as elements of vectors belonging to an observation space to be divided into $K$ classes $\left\{C_{k}\right\} k=1 \cdots K$. Given an observed feature vector $\mathbf{x}$, we want to determine the most probable class. More formally, we want to determine the class $C_{k}$ that maximizes the conditional probability $p\left(C_{k} / \mathbf{x}\right)$. Since classes are not directly observed, Bayes theorem is used to express $p\left(C_{k} / \mathbf{x}\right)$ in terms of the class-conditioned observation probability density $p\left(\mathbf{x} / C_{k}\right)$ in the form $p\left(C_{k} / \mathbf{x}\right)=p\left(\mathbf{x} / C_{k}\right) p\left(C_{k}\right) / p(\mathbf{x})$. Note that $p(\mathbf{x})$ is a scaling factor that is irrelevant to the maximization of $p\left(C_{k} / \mathbf{x}\right)$, and that a priori probability $p\left(C_{k}\right)$ 


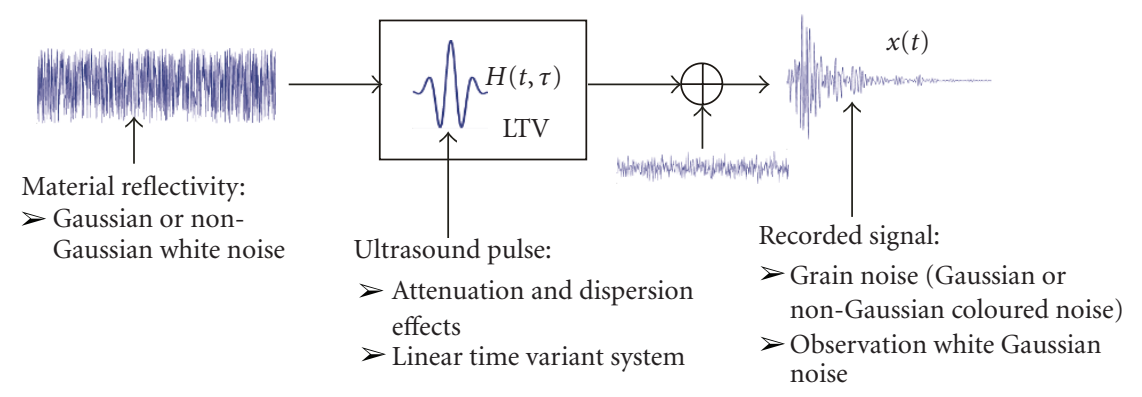

FIgURE 1: The through-transmission linear time variant model.

is assumed to be known (or equal to $1 / K$ for all classes). Hence, the key problem focuses on estimation of $p\left(\mathbf{x} / C_{k}\right)$. A nonparametric classifier tries to estimate $p\left(\mathbf{x} / C_{k}\right)$ from a training set of observation vectors, but this becomes progressively intractable as the dimension of the observation space (number of features) increases, because the required size of the training set becomes prohibitive. On the other hand, a parametric classifier assumes a given form for $p\left(C_{k} / \mathbf{x}\right)$ and, thus, tries to estimate the required parameters from the training observation set [9]. Most of the classifiers from parametric approaches consider Gaussian densities to simplify the problem in the absence of other information that could lead to better choices. Moreover, both parametric and nonparametric classifiers are very much complicated in semisupervised scenarios, that is, when part of the observed vectors belonging to the training set have unknown classes [10].

Therefore, procedures that would be of interest in the general area of classification should combine the following characteristics: the versatility of the nonparametric approach (from the point of view of the assumed form of $p\left(\mathbf{x} / C_{k}\right)$ ); the simplicity of the parametric methods (in the sense that most effort will concentrate on the estimation of a finite set of parameters); and operate in semisupervised scenarios. This is especially remarkable in the area of nondestructive classification of materials. On one hand, the prediction of the joint density of some selected features is almost impossible (Gaussianity is an assumption that is too restrictive in many cases). On the other hand, there are some applications where the available set of specimens used to obtain the training set can hardly be classified. This happens, for example, when the specimen cannot be destroyed to find the true inner state or when the definition of the $K$ classes is not clearly known a priori.

The classification application considered in this paper has the conditions necessary for verifying the usefulness of a versatile classifier that is capable of working with semisupervised training. Ceramic composition is assumed to be different in different historic and protohistoric periods, so there should be opportunities to classify the pieces from features derived from ultrasonic analysis. Nevertheless, exact modelling of the propagation of ultrasound in ceramic and statistical characterization of the features is a complex matter.
Hence, it is advisable not to assume particular parametric distributions (like normal density) in the design of the classifier. On the other hand, very often, the archaeologist does not know the period of all the available specimens that could be used to form the training set of observation: semisupervised training is a requirement in this application. Even more interesting is that the expert archaeologist can assign some probabilities of classes (ranging from 0 to 1 ) to part of the pieces of the training set, scenario that we will call probabilistic semisupervision (PSS). Most of the semisupervised classifiers are not capable of dealing with PSS, only if the assigned probabilities to the labelled feature vectors are 0 or 1 .

In this paper, we consider the application of a classification method based on the independent component analysis mixture model (ICAMM) [11-15]. ICAMM has the two required conditions: versatile modelling and the possibility of PSS training. In ICAMM, it is assumed that every class satisfies an independent component analysis (ICA) model $[16,17]$ : vectors $\mathbf{x}_{k}$ corresponding to a given class $C_{k} k=$ $1 \cdots K$ are the result of applying a linear transformation $\mathbf{A}_{k}$ to a (source) vector $\boldsymbol{s}_{k}$, whose elements are independent random variables, plus a bias or centroid vector $\mathbf{b}_{k}$, that is, $\mathbf{x}_{k}=\mathbf{A}_{k} \mathbf{s}_{k}+\mathbf{b}_{k} k=1 \cdots K$. This implies that the overall density of the observation vectors may be expressed as the mixture $p(\mathbf{x})=\sum_{k=1}^{K} p\left(\mathbf{x} / C_{k}\right) p\left(C_{k}\right)$ which gives the name to the model. Moreover, it also implies that $p\left(\mathbf{x} / C_{k}\right)=$ $\left|\operatorname{det} \mathbf{A}_{k}^{-1}\right| p\left(\mathbf{s}_{k}\right)$ where $\boldsymbol{s}_{k}=\mathbf{A}_{k}^{-1}\left(\mathbf{x}-\mathbf{b}_{k}\right)$. Thus, estimation of $p\left(\mathbf{x} / C_{k}\right)$ means estimation of $\mathbf{A}_{k}^{-1}=\mathbf{W}_{k}$, and $\mathbf{b}_{k}$ (like in a parametric method) plus estimation of $p\left(\mathbf{s}_{k}\right)$. However, this problem is simpler than the original one since the joint density of the elements of $\boldsymbol{s}_{k}$ can be expressed as the product of the marginals $p\left(s_{k}\right)=p\left(s_{k 1}\right) p\left(s_{k 2}\right) \cdots p\left(s_{k N}\right)$. Therefore, a very complex $N$-dimensional problem (where $N$ is the number of features) is broken down into $N$ onedimensional problems that are more tractable. Actually, many different types of densities can be assumed for the marginals, thus relaxing the Gaussianity constraint, and allowing a nonparametric estimation. In this sense, ICAMM can be considered a hybrid method that compiles the advantages of nonparametric and parametric models. There are a few references of the application of this method in NDT [18]. 
In summary, given one measured feature vector $\mathbf{x}$, the assigned class is given by

$$
\begin{aligned}
C(\mathbf{x}) & =\underbrace{\max }_{C_{k}} p\left(C_{k} / \mathbf{x}\right), \\
& =\underbrace{\max }_{C_{k}}\left|\operatorname{det} \mathbf{W}_{k}\right| p\left(\mathbf{s}_{k}\right) p\left(C_{k}\right), \quad k=1 \cdots K,
\end{aligned}
$$

where $\boldsymbol{s}_{k}=\mathbf{W}_{k}\left(\mathbf{x}-\mathbf{b}_{k}\right)$, and $\mathbf{W}_{k}, \mathbf{b}_{k}$ and $p\left(\mathbf{s}_{k}\right)$ are estimated by means of a PSS training. This is achieved using an iterative algorithm that we briefly describe below (a more detailed description can be found in [15]). A relevant concept in ICAMM learning is the embedded ICA algorithm. As ICAMM is a set of multiple ICA models, learning of the ICAMM parameters is essentially equivalent to simultaneous learning of a set of ICA parameters. Thus, any ICA algorithm could be used as part of the global ICAMM learning algorithm as we describe below.

Let us consider that the set of training feature vectors is formed by $\mathbf{x}_{m} m=1 \cdots M$. We divide the set into two subsets. The first subset is formed by $\mathbf{x}_{m} m=$ $1 \cdots M_{1} M_{1} \leq M$ vectors such that the expert archaeologist is capable of assigning some $p^{(0)}\left(C_{k} / \mathbf{x}_{m}\right)$ ranging between 0 and 1 for $k=1 \cdots K$. The second subset is formed by $M_{2}=M-M_{1}$ vectors where no knowledge exists about the possible class they belong to. The learning algorithms proceed in the following manner.

Initialization. For $k=1 \cdots K$, compute

$\mathbf{b}_{k}^{(0)}=\sum_{m=1}^{M_{1}} \mathbf{x}_{m} p^{(0)}\left(C_{k} / \mathbf{x}_{m}\right)$ (If $M_{1}=0$, then select the initial centroids randomly);

$\mathbf{W}_{k}^{(0)}$ (randomly);

$p^{(0)}\left(\mathbf{s}_{k}\right)$ (in a form depending on the selected embedded ICA algorithm) using $\mathbf{s}_{k m}^{(0)}=\mathbf{W}_{k}^{(0)}\left(\mathbf{x}_{m}-\mathbf{b}_{k}^{(0)}\right)$.

Updating. For $i=1 \cdots I$ and for $k=1 \cdots K$, compute

for the probabilistically labelled vectors

$$
p^{(i)}\left(\frac{C_{k}}{\mathbf{x}_{m}}\right)=p^{(0)}\left(\frac{C_{k}}{\mathbf{x}_{m}}\right) \quad m=1 \cdots M_{1} .
$$

For the unlabelled vectors

$$
\begin{aligned}
& p^{(i)}\left(C_{k} / \mathbf{x}_{m}\right)=\frac{p^{(i)}\left(C_{k} / \mathbf{x}_{m}\right) p\left(C_{k}\right)}{p\left(\mathbf{x}_{m}\right)}, \\
&=\frac{\left|\operatorname{det} \mathbf{W}_{k}^{(i-1)}\right| p^{(i-1)}\left(\mathbf{s}_{k m}^{(i-1)}\right) p\left(C_{k}\right)}{\sum_{k^{\prime}=1}^{K}\left|\operatorname{det} \mathbf{W}_{k^{\prime}}^{(i-1)}\right| p^{(i-1)}\left(\mathbf{s}_{k^{\prime} m}^{(i-1)}\right) p\left(C_{k^{\prime}}\right)}, \\
& m=M_{1}+1 \cdots M, \\
& \mathbf{b}_{k}^{(i)}=\sum_{m=1}^{M} \mathbf{x}_{m} p^{(i)}\left(\frac{C_{k}}{\mathbf{x}_{m}}\right), \\
& \mathbf{W}_{k}^{(i)}=\mathbf{W}_{k}^{(i-1)}+\Delta \mathbf{W}_{k}^{(i-1)}, \\
& \Delta \mathbf{W}_{k}^{(i-1)}=\sum_{m=1}^{M} \Delta \mathbf{W}_{k m(\text { ICA) }}^{(i-1)} p^{(i-1)}\left(\frac{C_{k}}{\mathbf{x}_{m}}\right) \\
&\left(\Delta \mathbf{W}_{k m(\text { ICA })}^{(i-1)} \text { is the updating due to training sample } \mathbf{x}_{m}\right. \\
& \text { in the selected embedded ICA algorithm). } \\
& p^{(i)}\left(\mathbf{s}_{k}\right) \text { (in a form depending on the selected embed- } \\
& \text { ded ICA algorithm) using } \mathbf{s}_{k m}^{(i)}=\mathbf{W}_{k}^{(i)}\left(\mathbf{x}_{m}-\mathbf{b}_{k}^{(i)}\right) .
\end{aligned}
$$

\section{Experiments and Results}

Two identical transducers (one in emitter mode and the other one in receiver mode) with a nominal operating frequency of $1.05 \mathrm{MHz}$ were used to obtain the throughtransmission signals. This operating frequency was selected after performing different tests, as the most appropriate to achieve small ultrasound attenuation with resolution enough to separate different kinds of ceramics. Sampling frequency was $100 \mathrm{MHz}$ and the observation time was $0.1 \mathrm{~ms}$ (10000 samples) for every acquisition. To reduce observation noise 16 acquisitions were averaged. The size of the transducers was also important since the ceramic pieces were small (a few centimetres in height and length, and less than one centimetre in width, see Figure 2).

The ceramic pieces were measured using a device where the ceramic piece is placed between two cases that adjust to the curved surfaces of the piece (see Figure 3 ). This device was implemented to perform controlled and repeatable measurements, thereby improving the manual recording. A rubber adaptor was used as coupling medium to match the acoustical impedance of the transducer to the piece. The adaptor has a good coupling to the surface of the material and be innocuous to the piece. The emitter is located in a case on the lower side of the piece and the receiver is located in case on the upper side of the piece. Note that the transducers are embedded into a case that has a pressure control that allows the force that is applied to the material to be the same for each measurement. Since the propagation velocity is an important feature for classification, the device has a mechanism that allows that piece thickness to be measured and transmitted to the signal processing system simultaneously with the ultrasound measurement.

The distribution of the pieces was: 47 Bronze Age, 155 Iberian, 138 Roman, and 140 Middle Ages. Thus, a total of 


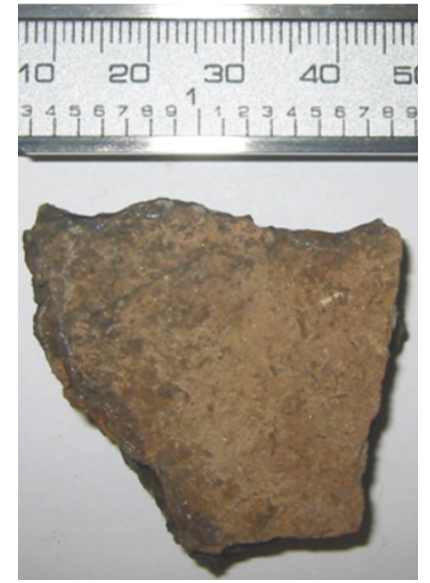

Bronze Age

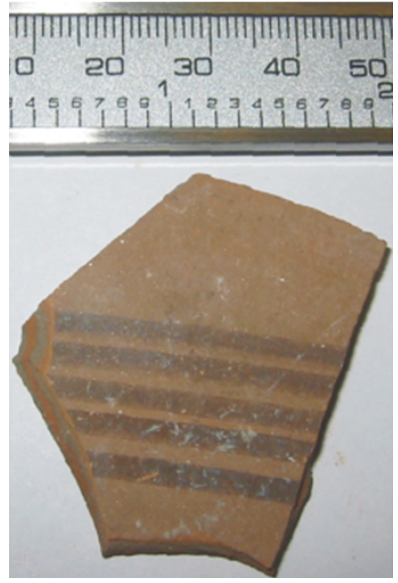

Iberian

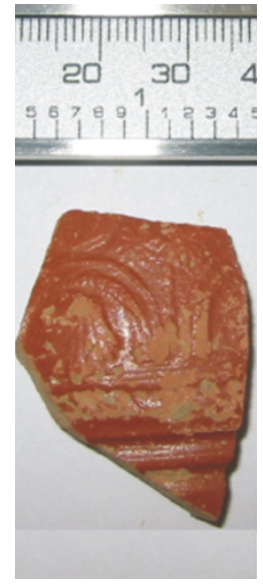

Roman

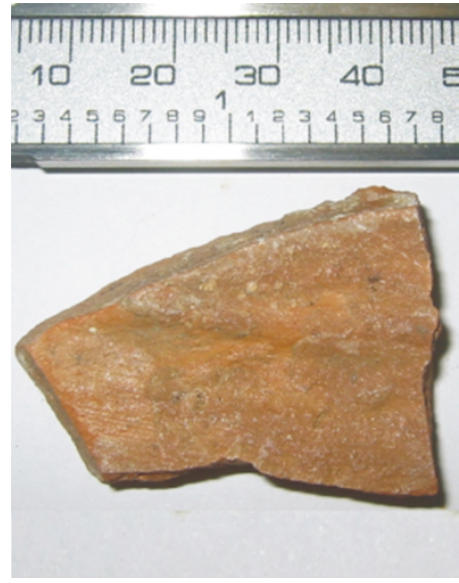

Middle Ages

FIGURE 2: Images of typical ceramic pieces.

480 pieces were used in the experiments from deposits at the Valencian Community in Spain. The features were selected from the features defined in Section 2. A total of 11 features were considered. The first 4 were the time averages over the whole acquisition interval of the 4 ultrasonic signatures defined in (1). The squared magnitude of the Short Term Fourier Transform was used to estimate $P_{x}(f, t)$.

Feature number 5 was $f_{c}\left(t_{0}\right)$, the instantaneous value of the centroid frequency at a specific time instant. The parameters $A, \beta, P, H O M$, and $T R$ that are defined in Section 2, were also included in the feature vector. Finally, the velocity of propagation $v$ of the ultrasound, which was measured by dividing the piece thickness by the pulse arrival delay, was also considered, since it is a standard variable in the ultrasonic characterization of materials. Figure 4 shows examples of the time record, spectrum, and histogram for each period. It also shows the eleven features obtained for each example. Note the significant differences (in general) among the feature values corresponding to different periods, which provide the opportunity for good classification performance.

First, the signal features were preprocessed with Principal Component Analysis (PCA) [19] to reduce the dimension of the problem as much as possible and to detect redundancies among the selected features. This resulted in only 6 significant features (components), which were linear combinations of the original ones. These 6 components explained a total of $90 \%$ of the data variance.

We had a total of $480 * 0.75=360$ original samples for training. By adding spherical Gaussian noise to the original samples, three replicates were estimated to obtain a total of 1440 samples for training. We performed 100 runs varying the sets of 360 samples used for training and 120 used for testing. The percentage of success in determining the correct class was then evaluated for a total of $120 \times 100$ testing samples.

Different alternative ICAMM classifiers were implemented together with other classical classifiers. We considered four embedded ICA algorithms: nonparametric ICA
TABle 1: Classification accuracy (percentage) obtained with the different variants of ICAMM.

\begin{tabular}{lcccc}
\hline PSS ratio & NP-ICA & JADE & TDSEP & fastICA \\
\hline 1 & 0.83 & 0.81 & 0.79 & 0.81 \\
0.8 & 0.79 & 0.75 & 0.69 & 0.71 \\
0.6 & 0.72 & 0.67 & 0.66 & 0.60 \\
0.4 & 0.65 & 0.64 & 0.55 & 0.59 \\
\hline
\end{tabular}

TABLE 2: Classification accuracy (percentage) obtained with the other methods.

\begin{tabular}{lllcc}
\hline LDA & RBF & LVQ & MLP & $k N N$ \\
\hline 0.73 & 0.64 & 0.59 & 0.67 & 0.64 \\
\hline
\end{tabular}

(NP-ICA) [15, 20]; JADE [21]; TDSEP [22]; and fastICA [23]. Several PSS ratios were also tested (PSS ratio is defined as the proportion between probabilistically labelled and unlabelled data in the training stage). Linear Discriminant Analysis (LDA) classifier [19] was also verified as it is representative of a supervised classifier optimum under Gaussianity assumptions. Some other classifiers based on neural networks schemes were also implemented: Radial Basis Function (RBF), Learning Vector Quantization (LVQ), and Multilayer Perceptron (MLP) [24]. As well the $k$-nearest neighbor $(k N N)$ was tested [19].

Table 1 shows the overall percentage of classification accuracy achieved by the different ICAMM variants.

Table 2 shows the overall percentage of classification accuracy achieved by the other different methods implemented. Note that different values of the fitting variables required in each method (e.g., the value $k$ in $k \mathrm{NN}$ ) were tested and the results shown are the best ones obtained.

The best performance in classification was obtained using ICAMM NP-ICA at PSS ratio of 1 (total probabilistic supervision), achieving a classification accuracy of $83 \%$, which is much better than the rest of supervised methods (LDA, RBF, LVQ, MLP, and $k \mathrm{NN}$ ). As the PSS ratio in 


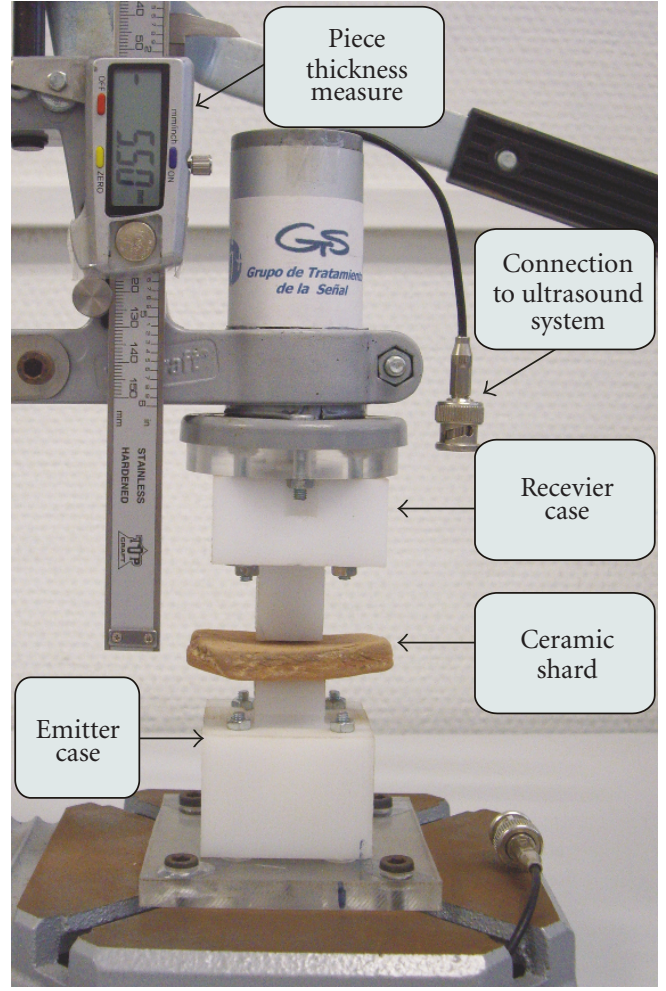

(a)

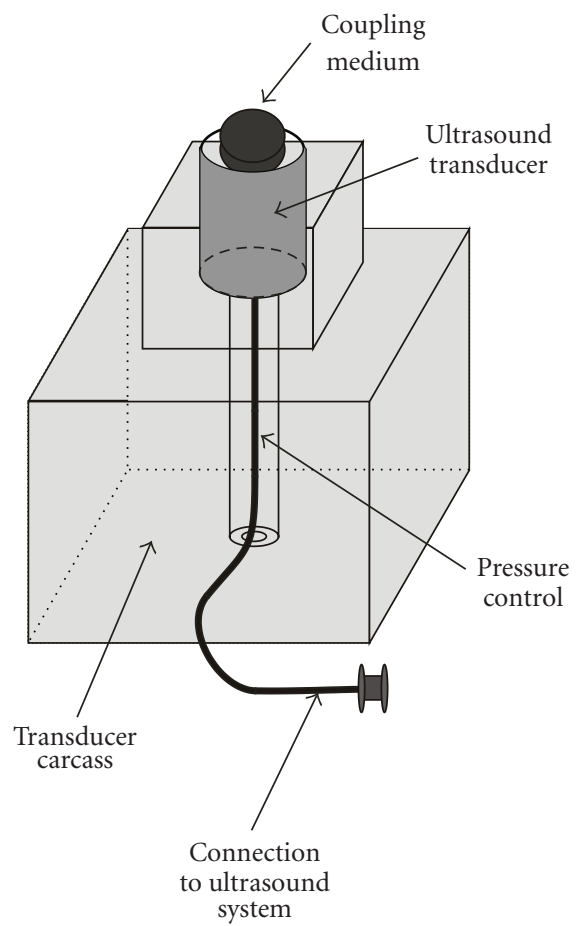

(b)

Figure 3: Measurement device employed in ultrasonic signal acquisition. A detail of the ultrasound transducer case is included.

ICAMM is reduced, the performance gets worse. However, for PSS ratio 0.8, ICAMM NP-ICA is still the best one with classification accuracy of $79 \%$. For PSS ratio 0.6, only LDA gives a slightly better result. This confirms the convenience of not assuming any parametric model of the underlying probability density as is assumed in LDA and in the parametric ICAMM variants. Besides, other supervised nonparametric methods (RBF, LVQ, MLP, and $k \mathrm{NN}$ ) cannot compete with NP-ICA since it is a hybrid method with an implicit parametric model (ICA), which allows of a training set of relatively small size.

To gain more insight into the classifier performance, we include Table 3 , which contains the confusion matrix obtained by NP-ICA for 1 PSS ratio. The Roman and Iberian categories are not very difficult to classify, but they are often confused with each other. The pieces from the Middle Ages are confused with Bronze Age pieces 14\% of the time, and Roman pieces cause misclassification of some pieces from the Bronze Age and the Middle Ages.

\section{Discussion}

In order to draw a physical interpretation of the results obtained by ultrasounds, a diversity of morphological and physiochemical characterization analyses were carried out using conventional instrumental techniques. A stratified random sampling analysis was made using data from the physical analysis of the pieces: open porosity and apparent
TABLe 3: Confusion matrix (percentages) by NP-ICA with 1 PSS ratio.

\begin{tabular}{lcccc}
\hline & Bronze Age & Iberian & Roman & Middle Ages \\
\hline Bronze Age & 0.79 & 0 & 0.07 & 0.14 \\
Iberian & 0 & 0.89 & 0.09 & 0.02 \\
Roman & 0.05 & 0.19 & 0.69 & 0.07 \\
Middle Ages & 0.02 & 0 & 0.05 & 0.93 \\
\hline
\end{tabular}

density $[25,26]$. Thus, a sample of the ceramic pieces for the different periods was obtained. The raw material composition of the selected pieces was analyzed using optical microscope and scanning electron microscope (SEM) $[27,28]$; and also the processing methods of the pieces were studied. From those analyses, the differences of the ceramic physical properties for the different periods and the ultrasound propagation are discussed.

5.1. Open Porosity and Apparent Density. A sample of the pieces was selected for morphological and physiochemical characterization based on open porosity and apparent density analyses of the pieces. For stratified random sampling, the values of these physical properties for the different periods were considered as random variables that follow Gaussian distribution. First, an estimation of the variable variance for the different periods (statistical strata) was made. This estimation was obtained from 45 representative 

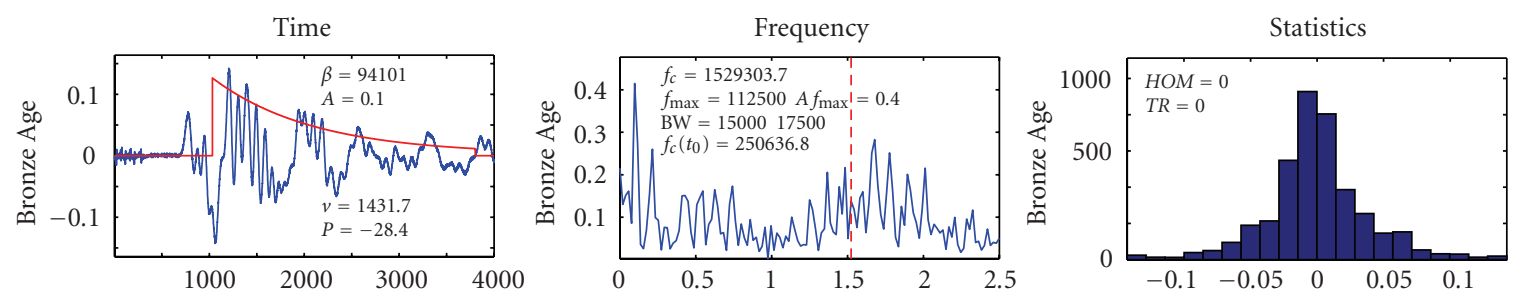

(a)
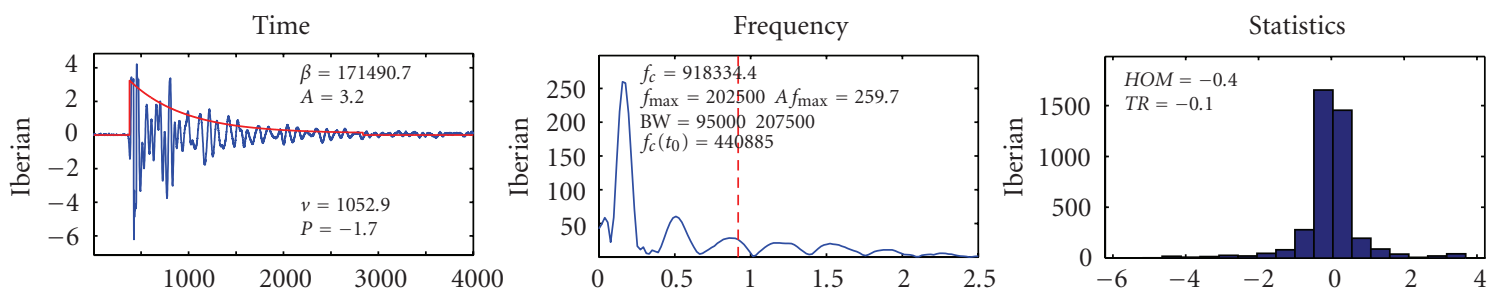

(b)
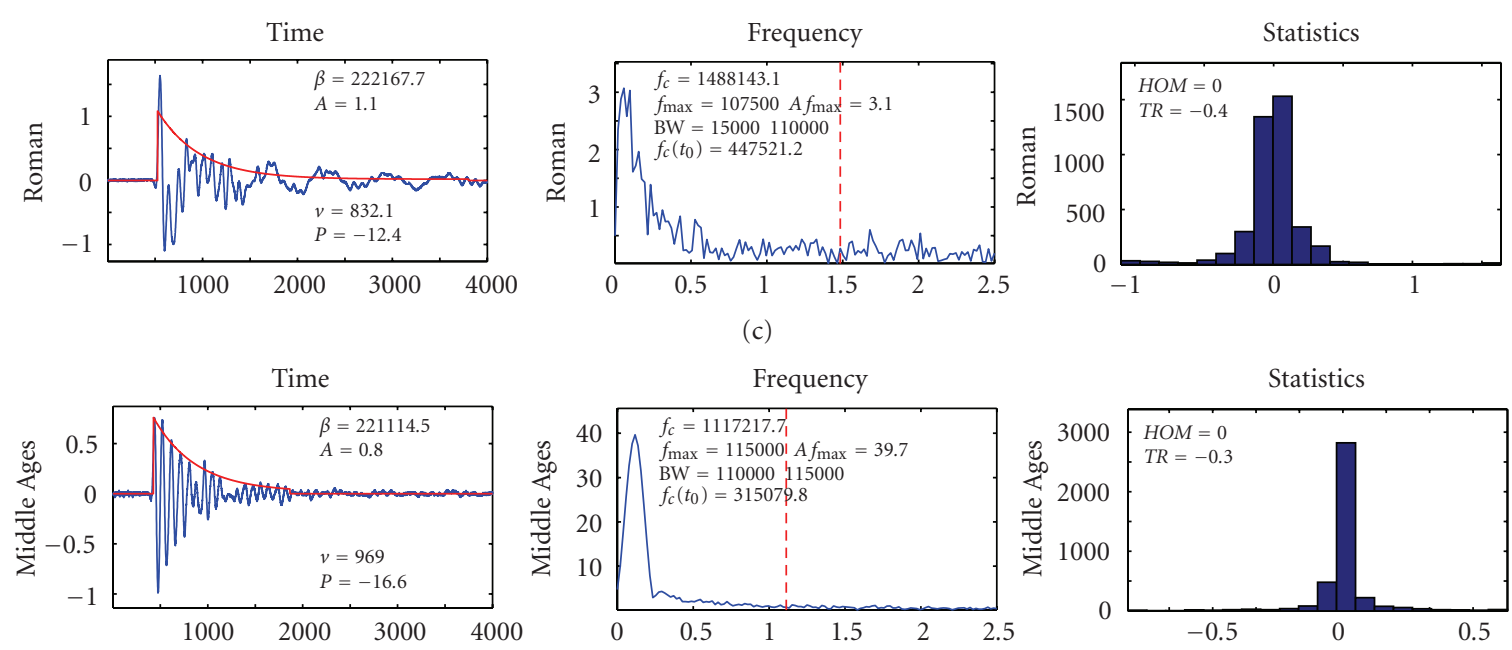

(d)

FIGURE 4: Some examples of time signals, spectra, histograms, and corresponding features extracted from ultrasonic signals for archaeological ceramic pieces from different periods. Units are: Time axis: sample number; Frequency axis: MHz; Statistics axis: bins of signal values; $P$ $(\mathrm{dB}) ; v(\mathrm{~m} / \mathrm{s}) ; f_{c}, f_{\max }, \mathrm{BW}, f_{c}\left(t_{0}\right)(\mathrm{Hz})$.

TABLe 4: Porosity and density statistics of the prior study.

\begin{tabular}{lcccc}
\hline & \multicolumn{2}{c}{ Open porosity (\%) } & \multicolumn{2}{c}{ Apparent density $\left(\mathrm{gr} / \mathrm{cm}^{3}\right)$} \\
Period $i$ & Mean $\hat{\mu}$ & $\begin{array}{c}\text { Standard } \\
\text { deviation } \hat{\sigma}_{i}\end{array}$ & Mean $\hat{\mu}$ & $\begin{array}{c}\text { Standard } \\
\text { deviation } \hat{\sigma}_{i}\end{array}$ \\
\hline (1) Bronze Age & 28.20 & 3.7794 & 1.80 & 0.0676 \\
(2) Iberian & 22.70 & 3.3320 & 1.85 & 0.0663 \\
(3) Roman & 31.06 & 8.3532 & 1.79 & 0.1607 \\
(4) Middle Ages 22.69 & 5.3441 & 1.84 & 0.0949 \\
\hline
\end{tabular}

pieces that were physically tested for open porosity and apparent density. The results of this prior study are shown in Table 4.

The objective of the sampling was to provide estimators with small variances at the lowest cost possible (considering that morphological and physiochemical characterization are costly). To estimate the fraction of the total sample size $n$ corresponding to the stratum $i$, we applied the so-called Neyman allocation [29], $n_{i} / n=N_{i} \sigma_{i} /\left(\sum_{i=1}^{L} N_{i} \sigma_{i}\right)$, where $L$ is the number of strata ( 4 periods for this application), $N_{i}$ is the sample number in the stratum $i(3,15$, and 15,12 for Bronze Age, Iberian, Roman, and Middle Ages pieces, respectively), and $\sigma_{i}$ is the standard deviation for the stratum $i$ (estimates of Table 4 were applied). The results for the strata $i=1 \cdots 4$ were: $n_{1} / n=6.85 \%, n_{2} / n=19.90 \%, n_{3} / n=44.42 \%$, and $n_{4} / n=28.83 \%$ for open porosity; and $n_{1} / n=6.5 \%$, $n_{2} / n=21.01 \%, n_{3} / n=45.34 \%$, and $n_{4} / n=27.16 \%$ for apparent density, respectively.

We specified that the estimate of the sample mean should lie between $B$ units of the population mean, with probability equal to .95. This is equivalent to impose that the mean estimate should lie in the interval $\mu \pm 2 \cdot \sigma$, that is, $B=2 \cdot \sigma$. From the analysis of the variable means of Table 4 , we chose $B=1.1 \%$ and $B=0.02 \mathrm{gr} / \mathrm{cm}^{3}$ as the bounds on the error of estimation of the population mean for open porosity 


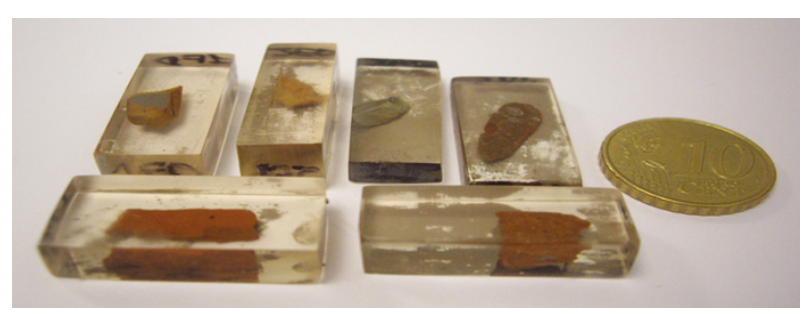

FIGURE 5: Bits taken from the ceramic fragments included in the test probes prepared for the Scanning Electron Microscope.

and apparent density, respectively. These bounds allowed the stratum mean of the sampling to be separated adequately.

The total number of samples was estimated using [29], $n=\left(\sum_{i=1}^{L} N_{i} \sigma_{i}\right)^{2} /\left(N^{2} \cdot D+\sum_{i=1}^{L} N_{i} \sigma_{i}^{2}\right)$, where $D=B^{2} / 4$. Thus, we obtained the total number of samples $n=79$ and $n=83$ for open porosity and apparent density, respectively. These were the number of pieces that the morphological and physiochemical characterization analyses were applied to. Using the estimated fractions $n_{i} / n$ for the strata and the total number of samples $n$, we obtained the sampling population for each stratum. The final results of the stratified random sampling for an error margin of .05 are in Table 5. The estimate of the population mean for open porosity and apparent density for each stratum are shown with an approximate 2 standard deviation bound on the error of estimation.

Table 5 shows that the samples of the different strata (chronological periods) can be clearly separated by open porosity, since the bounds of the distributions define the most part of the densities to be disjoint. The separation of the samples by apparent density is more difficult because there is a degree of overlapping between densities of Roman and Bronze Age pieces, and a higher overlapping between densities of Iberian and Middle Ages pieces. However the joint densities of these two collections of pieces are wellseparated between them. In conclusion, physical properties of the ceramics shows that it is possible a separation of the pieces in the different chronological periods of this study. Different porosities and densities of the pieces are determined by the material composition and processing methods employed in the ceramic manufacturing. These issues are studied in the next section.

5.2. Ceramic Composition and Processing. The selected pieces were observed, photographed, and then analyzed using an optical microscope and a scanning electron microscope (SEM). Some of the test tubes prepared for SEM are shown in Figure 5.

The data provided by optical microscope and SEM show that there are clear differences at a morphological level between the different groups of processed fragments. Therefore, the ceramic pieces corresponding to the Bronze Age exhibited a dark brown tone and the presence of a lot of dark-toned ferrous-composition spots that are associated with magnetite as well as reddish ferrous iron oxide nuclei. The Iberian ceramic pieces had varying shades between orange and black. The quartz temper was big or very big grains and abundant ferrous iron oxide nuclei as well as more isolated dark magnetite spots were found. This was an iron-rich ceramic (up to $7.45 \%$ of $\mathrm{Fe}_{2} \mathrm{O}_{3}$ ) with a high content of calcium (up to $6.30 \%$ of $\mathrm{CaO}$ ). The fragments of Roman ceramic had variable characteristics depending on the typology (sigillata, common, and amphora). In any of these, the pieces were made of an orange-toned paste with small-size porosity and small quantity of temper that increased from the amphora to the sigillata typology. Roman ceramic showed content of $\mathrm{Fe}_{2} \mathrm{O}_{3}$ of $5.71 \%, 6.36 \%$ and $9.24 \%$, and content of $\mathrm{CaO}$ of $0.67 \%, 2.92 \%$ and $1.29 \%$ for sigillata, common, and amphora, respectively. Finally, the ceramic from the Middle Ages had a bright orange to brown colour that indicates they are made of ferrous paste. This ceramic contains abundant small to very small nuclei of red ferrous iron oxide as well as dark-toned magnetic spots and quartz temper of big or very big grains. Also, limestone aggregates of white tone associated with high content of $\mathrm{CaO}$ (around 8\%) were observed.

With regard to the methods used to manufacture the ceramics, they were different according to the evolution in time of the processing techniques. The set of ceramic fragments were from three regions (Requena, Enguera, and Liria) from the Valencia Community at the East of Spain. The pieces of the Bronze Age were from Requena (XXX-XX centuries B.C.). They were handmade using basic appliances, with an appearance very coarse, rudimentary, and of irregular texture for household. Manufacturing was local and authentic of every town; it was related to the women's domestic activities. From the dark tone of the Bronze Age ceramics, it can be inferred that they were made in reducing atmosphere, that is, in closed oven at low temperatures. Iberian fragments corresponded to brushdecorated with geometric, zoomorphic, and human motifs or nondecorated vessels. These pieces have been dated at about V-III centuries B.C and they were from three different deposits. Paste of the Iberian ceramics was much more fine and elaborated than the Bronze Age ceramic paste. The technological innovation in the processing of the pieces was the use of lathe.

The Roman fragments of the three groups (sigillata, common, and amphora) showed technical perfection of manufacture using different techniques: lathe, handmade, and mold. They were from I-III centuries. In this period, the applications of molds for potters allowed mass production of ceramics. Sigillata ceramic features a red bright varnish that is obtained applying a clay solution to the ceramic surface and cooking at high temperatures in open oven (oxidizing atmosphere). Sigillata pieces were decorated with reliefs of different motifs and were luxury ceramic. Common and amphora types of Roman ceramic were made using lathe. They were rough appearance without decoration and for household and/or storage or transport use. The Middle Ages pieces were of two subperiods: Islamic and Christian (around VIII-X centuries). The Islamic pieces were from caliphate vessels of paste simple elaborated without decoration and special treatment. The Christian pieces were white gross paste of diverse typologies, some without decoration and 
TABLE 5: Statistics of the stratified random sampling for open porosity and apparent density.

\begin{tabular}{llccccc}
\hline & & $N_{i}$ & $n_{i}$ & $\mu_{i}$ & $\mu_{i} \mp 2 \cdot \sqrt{\left(\left(N_{i}-n_{i}\right) / N_{i}\right)\left(\sigma_{i}^{2} / n_{i}\right)}$ & 30,90 \\
\hline \multirow{5}{*}{ Open porosity } & (1) Bronze Age & 47 & 5 & 29,30 & 27,70 & 23,29 \\
& (2) Iberian & 155 & 16 & 22,50 & 21,71 & 33,22 \\
& (3) Roman & 138 & 35 & 32,00 & 22,78 & 24,82 \\
\hline \multirow{5}{*}{ Apparent density } & (4) Middle Ages & 140 & 23 & 23,80 & 1,78 & 1,88 \\
& (1) BronzeAge & 47 & 5 & 1,85 & 1,75 & 1,89 \\
& (2) Iberian & 155 & 17 & 1,77 & 1,85 & 1,80 \\
\hline
\end{tabular}

some with incisions or decorations in black painted with manganese oxide.

\subsection{Ceramic Physical Properties and Ultrasound Propagation.} The differences in physical properties, composition and processing of the ceramic pieces, presented above, suggest the possibility of devising nondestructive techniques for archaeological ceramic classification. In Section 5.1, it was shown that the pieces could be separated by chronological periods using measures of their open porosity and apparent density. Besides, it is well-known that porosity and density of a material have a definite influence on the propagation of the ultrasound $[30,31]$. Thus, it is clear that should be there correlation between the results obtained by the proposed method based on ultrasounds (Section 4) and the differences in physical properties of the pieces for the different chronological periods.

There are several factors that can determine the porosity and density of ceramics, such as the raw material composition and the processing method employed to manufacture the pieces. However, in the case of archaeological ceramics, the original ceramic physical properties after manufacturing, can be altered by other factors such as the ceramic use (i.e., over-heating for cooking, etc.) and in general with the pass of the time (i.e., fractures, loss of cover layers, etc.). Thus, an exhaustive analysis of physical properties and how these properties were derived for archaeological ceramics becomes a very complex problem that needed an important amount of information that is outside the scope of this work. Note that the objective of this work is to provide a new NDT procedure to classify archaeological ceramics from the basis of training with a set of pieces of known class made with the intervention of an expert. A correct training will determine the achievement of the procedure to classify ceramics of unknown class.

The analysis of the results obtained by ultrasounds provided here consider correct (or at least probabilistic) labelling made by the expert and are based on available data of the composition, processing and physical features of the ceramics shown in Sections 5.1 and 5.2. Let us explain the misclassifications in the confusion matrix of the ultrasoundbased classification of Table 3. Misclassification is obtained from similar responses of pieces from different periods to the ultrasounds. Table 3 shows that Roman ceramics is the most misclassified group. Confusion between Roman and
Iberian pieces (19\% and 9\%) can be explained from ceramic composition and processing. The amphora and common Roman pieces were made from iron-rich paste and using lathe as well as the Iberian pieces. Thus, the mechanical and physical properties for these two groups were similar.

The confusion between Roman and Bronze Age pieces (5\% and $7 \%$ ) can be explained due to changes in the structure of some of the Roman pieces of the sigillata subgroup that had lost the cover varnish. The high value of porosity shown by the fragments of sigillata is associated with pores of very small-size and very connected, which allows big water absorption once the varnish is removed. Thus, these two groups of pieces show similar physical properties due accidents cause with the pass of the time. Regarding to the confusion between Bronze Age and Middle Ages pieces (14\% and $2 \%)$, this also can be explained from composition and processing. The Islamic subgroup of Middle Ages pieces were from the "paleoandalusî" period (early centuries of the Islamic period in Spain). During, this period, the productive strategy of household chose intentionally to simplify the production process. Simple ways of ceramic manufacture and cooking were employed to obtain kitchen's recipients with thermal shock resistance. Thus, ceramics were manually made from little-decanted clays and cooked at low temperatures. The results were coarse pieces from the Middle Ages with physical properties comparable to the Bronze Age pieces [32].

Let us analyze the ultrasound-based results from the point of view of the porosity and density. We observed that the porosity and density of the Bronze Age pieces are relatively close to porosity and density of the pieces from Roman and Middle Ages. This explains why $7 \%$ and $14 \%$ of the Bronze Age pieces were assigned to the Roman and Middle Ages periods in Table 3. Similarly, the pieces from the Iberian period and the Middle Ages have similar porosities and densities, so this may justify why $2 \%$ of the Iberian pieces were assigned to the Middle Ages.

The $9 \%$ of pieces of the Iberian period that should have been assigned to the Roman period were incorrectly assigned because the Iberian ceramic is very close to one of the three kinds of Roman ceramics (sigillata, common, and amphora)-the common kind-. This also explains why the corresponding 19\% of pieces of the Roman period were incorrectly assigned to the Iberian period. No clear explanation exists for the lack of symmetry in the confusion 
matrix of Table 3; however, it must be taken into account that the training process introduced some degree of arbitrariness because of the probabilistic labelling of the expert. Thus, it seems that the expert was able to clearly identify the pieces from Iberian and Middle Ages, but had more difficulties with the Bronze Age and Roman ones. This uncertainty may have been transmitted to the classifier during the training stage.

The experiments with classical methods of ceramic characterization used in archaeology not only show that correlations between the extracted parameters from the ultrasound signals and the physical properties of the materials were found. Moreover, they also have demonstrated some advantages of the proposed ultrasound method. The equipment required for nondestructive evaluation by ultrasound is, in general, less costly, and the experiments are easier to perform. The pieces are not damaged in any way during testing, nor is it necessary to alter or destroy any of the material that is analyzed. Very significant differences for the time required to analyze the pieces were demonstrated: the ultrasound analysis (measuring, processing, and automatic classification) for 480 pieces took only 6 hours; the SEM analysis (tube preparation and electron microscope analysis) for 80 pieces took 274 hours; the porosity and density analyses (immersion and weighing of the pieces) for 80 pieces took 288 hours.

There are limitations to the application of this procedure due to the fact that the training of the classifier must be performed from a specific set of data. Thus, the classifier must be adapted to a specific data model and its efficiency is restricted by the fact that the new data to be classified must follow the same data model. Nevertheless, the training of the classifier could progressively be improved by increasing the number of pieces for each known chronological period. With proper training, the classifier would be able to provide a prediction of the chronological period for pieces that do not have clear chronological markers. In addition, the semisupervised training mode could be used to model the uncertainty that expert archaeologists may have about the chronological period to which the pieces belong.

\section{Conclusions}

We have presented the results of applying ICAMM to a challenging application in the area of nondestructive testing of materials: the classification of archaeological ceramic pieces into different historic periods. We have demonstrated the interest of using methods that are able to consider nonGaussian models of the underlying probability densities in the feature vector space. Thus, an ICAMM classifier was tested using different variants depending on the embedded ICA algorithm. ICAMM has the additional merit of allowing PSS labelling, which is of practical interest in the considered application. Note that in any ICAMM variant, the mutual dependence among features is modelled in a parametric form; also note that in nonparametric ICAMM, the estimated marginals are nonparametric. This confirms that nonparametric ICAMM shares the good general modelling capability of nonparametric classifiers and also can work with a training set of relatively small size, which is a relevant property of parametric techniques. This explains the fact that nonparametric ICAMM has shown the best results and is able to produce acceptable performance for even low ratios of PSS.

The experiments show promising results in defining a standardised method that could complement or replace destructive, costly, and time-consuming techniques, which are currently being used by archaeologists in the area of ceramic characterization. Extensions of the procedures presented in this work to other emergent material applications are planned for future work.

\section{Acknowledgments}

This paper has been supported by the Spanish Administration and the FEDER Programme of the European Community under Grant TEC 2008-02975; the Generalitat Valenciana under Grant GV-ACOMP/2009/340, and Grant PROMETEO/2010/040.

\section{References}

[1] R. E. Taylor and M. J. Aitken, Chronometric Dating in Archaeology, vol. 2 of Advances in Archaeological and Museum Science Series, Springer, New York, NY, USA, 1997.

[2] R. Cribbs and F. Saleh, "An ultrasonic based system used for non-destructive imaging of archaeological sites," in Proceedings of Informatica ed Egittologia all'Inizio Degli Anni '90, pp. 97-108, Rome, Italy, 1996.

[3] A. Murray, M. F. Mecklenburg, C. M. Fortenko, and R. E. Green, "Detection of delaminations in art objects using aircoupled ultrasound," in Proceedings of Materials Issues in Art and Archaeology III Symposium, pp. 371-378, San Francisco, Calif, USA, 1992.

[4] W. I. Sellers and A. T. Chamberlain, "Ultrasonic cave mapping," Journal of Archaeological Science, vol. 25, no. 9, pp. 867873, 1998.

[5] A. Salazar, R. Miralles, A. Parra, L. Vergara, and J. Gosalbez, "Ultrasonic signal processing for archaeological ceramic restoration," in Proceedings of the IEEE International Conference on Acoustics, Speech and Signal Processing (ICASSP '06), pp. III1160-III1163, Toulouse, France, May 2006.

[6] L. Cohen and P. Loughlin, Recent Developments in TimeFrequency Analysis, Springer, New York, NY, USA, 1998.

[7] R. Miralles, L. Vergara, and J. Gosàlbez, "Material grain noise analysis by using higher-order statistics," Signal Processing, vol. 84, no. 1, pp. 197-205, 2004.

[8] R. Miralles, L. Vergara, A. Salazar, and J. Igual, "Blind detection of nonlinearities in multiple-echo ultrasonic signals," IEEE Transactions on Ultrasonics, Ferroelectrics, and Frequency Control, vol. 55, no. 3, pp. 637-647, 2008.

[9] A. K. Jain, R. P. W. Duin, and J. Mao, "Statistical pattern recognition: a review," IEEE Transactions on Pattern Analysis and Machine Intelligence, vol. 22, no. 1, pp. 4-37, 2000.

[10] O. Chapelle, B. Schölkopf, and A. Zien, Semi-Supervised Learning, MIT Press, Cambridge, Mass, USA, 2006.

[11] T.-W. Lee, M. S. Lewicki, and T. J. Sejnowski, "ICA mixture models for unsupervised classification of non-Gaussian classes and automatic context switching in blind signal separation," 
IEEE Transactions on Pattern Analysis and Machine Intelligence, vol. 22, no. 10, pp. 1078-1089, 2000.

[12] R. A. Choudrey and S. J. Roberts, "Variational mixture of Bayesian independent component analyzers," Neural Computation, vol. 15, no. 1, pp. 213-252, 2003.

[13] C.-T. Lin, W.-C. Cheng, and S.-F. Liang, "An on-line ICA-mixture-model-based self-constructing fuzzy neural network," IEEE Transactions on Circuits and Systems I, vol. 52, no. 1, pp. 207-221, 2005.

[14] C. A. Shah, P. K. Varshney, and M. K. Arora, "ICA mixture model algorithm for unsupervised classification of remote sensing imagery," International Journal of Remote Sensing, vol. 28, no. 8, pp. 1711-1731, 2007.

[15] A. Salazar, L. Vergara, A. Serrano, and J. Igual, "A general procedure for learning mixtures of independent component analyzers," Pattern Recognition, vol. 43, no. 1, pp. 69-85, 2010.

[16] A. Hyvärinen, J. Karhunen, and E. Oja, Independent Component Analysis, John Wiley \& Sons, New York, NY, USA, 2001.

[17] P. Comon, "Independent component analysis-a new concept?” Signal Processing, vol. 36, no. 3, pp. 287-314, 1994.

[18] A. Salazar, L. Vergara, and R. Llinares, "Learning material defect patterns by separating mixtures of independent component analyzers from NDT sonic signals," Mechanical Systems and Signal Processing, vol. 24, no. 6, pp. 1870-1886, 2010.

[19] R. O. Duda, P. E. Hart, and D. G. Stork, Pattern Classification, Wiley-Interscience, New York,NY, USA, 2nd edition, 2000.

[20] R. Boscolo, H. Pan, and V. P. Roychowdhury, "Independent component analysis based on nonparametric density estimation," IEEE Transactions on Neural Networks, vol. 15, no. 1, pp. 55-65, 2004.

[21] J. F. Cardoso and A. Souloumiac, "Blind beamforming for non-Gaussian signals," IEE Proceedings, Part F, vol. 140, no. 6, pp. 362-370, 1993.

[22] A. Ziehe and K. R. Müller, "TDSEP- an efficient algorithm for blind separation using time structure," in Proceedings of the 8th International Conference on Artificial Neural Networks (ICANN '98), pp. 675-680, Skövde, Sweden, September 1998.

[23] A. Hyvärinen, "Fast and robust fixed-point algorithms for independent component analysis," IEEE Transactions on Neural Networks, vol. 10, no. 3, pp. 626-634, 1999.

[24] C. M. Bishop, Neural Networks for Pattern Recognition, Oxford University Press, Oxford, UK, 2004.

[25] P. M. Rice, Pottery Analysis: A Sourcebook, The University of Chicago Press, Chicago, Ill, USA, 1989.

[26] K. G. Harry and A. Johnson, "A non-destructive technique for measuring ceramic porosity using liquid nitrogen," Journal of Archaeological Science, vol. 31, no. 11, pp. 1567-1575, 2004.

[27] A. M. Pollard and C. Heron, Archaeological Chemistry, The Royal Society of Chemistry, Cambridge, UK, 2008.

[28] S. L. Olsen, Scanning Electron Microscopy in Archaeology, British Archaeological Reports, Institute of Physics, Oxford, UK, 1998.

[29] S. K. Thompson, Sampling, Wiley-Interscience, New York, NY, USA, 2nd edition, 2002.

[30] J. D. Cheeke, Fundamentals and Applications of Ultrasonic Waves, CRC Press, LLC, Boca Raton, Fla, USA, 2002.

[31] J. Krautkrämer, Ultrasonic Testing of Materials, Springer, Berlin, Germany, 4th edition, 1990.

[32] M. Alba-Calzado and S. Gutiérrez-Lloret, "Las producciones de transición al Mundo Islámico: el problema de la cerámica paleoandalusí (siglos VIII y IX)," in Cerámicas Hispanorromanas: Un Estado de la Cuestión, B. Casasola and A. Ribera i Lacomba, Eds., pp. 585-613, Universidad de Cadiz, Cádiz, Spain, 2008. 